\title{
Fatores associados à busca por serviços preventivos de saúde entre adultos brasileiros: Pesquisa Nacional de Saúde, 2013
}

\author{
Factors associated with preventive health services search among \\ Brazilian adults: National Health Survey, 2013
}

Silvia Lanziotti Azevedo da Silva (https://orcid.org/0000-0002-2323-2029) ${ }^{1}$

Juliana Lustosa Torres (https://orcid.org/0000-0002-3687-897X) ${ }^{2}$

Sérgio Viana Peixoto (https://orcid.org/0000-0001-9431-2280) ${ }^{3}$

${ }^{1}$ Instituto de Ciências da Motricidade, Universidade Federal de Alfenas. Av. Jovino Fernandes Sales 2600, Santa Clara. 31270901 Alfenas MG Brasil. silviafisiojf@yahoo.com.br

${ }^{2}$ Medicina Preventiva e Social, Universidade Federal de Minas Gerais. Belo Horizonte MG Brasil ${ }^{3}$ Centro de Pesquisas René Rachou, Fiocruz Minas. Belo Horizonte MG Brasil

\begin{abstract}
The objective of this article is to examine factors associated with preventive health services search among Brazilian adults. Sample included adults participants from the National Health Survey (2013), that had reported any health service search in prior 15 days, categorized into "treatment/diagnosis" or "preventive" service. Exploratory variables included sex, age, race, marital status, education, household situation, private health plan enrolment and time of FHS enrolment. Associations were verified by prevalence ratios $(P R)$, estimated using robust Poisson regression, considering complexity of sampling parameters. Final sample included 32,377 individuals, 12,94\% have searched "preventive" services. Preventive search was more often among women and less often among the older adults, those not living with partner, with less education. Having private health plan was associated with less preventive services search. FHS enrolment were not associated with preventive search. By conclusion, although some efforts and the importance of preventive actions, most of individuals search for treatment services.
\end{abstract}

Key words Primary preventive healthcare, Health services, Family health strategy, Prepaid health plans, Epidemiological surveys.
Resumo $O$ objetivo deste artigo é verificar os fatores associados à busca por serviços preventivos de saúde pela população adulta brasileira. A amostra foi composta por participantes da Pesquisa Nacional de Saúde (2013), que relataram procura por algum serviço de saúde nos últimos 15 dias, classificado em "tratamento/diagnóstico" ou "preventivo". As variáveis exploratórias incluíram sexo, faixa etária, cor de pele, estado conjugal, escolaridade, localização do domicílio, posse de plano privado de saúde e tempo de cadastro na ESF. As associações foram verificadas pelas razões de prevalência (RP), obtidas pelo modelo de Poisson, considerando a complexidade do desenho amostral. A amostra foi composta por 32.377 indivíduos que buscaram o serviço de saúde, sendo 12,94\% para ações "preventivas”. A procura por estes cuidados foi mais frequente entre mulheres $e$ menos frequente entre os mais velhos, que não viviam com companheiro, com menor escolaridade. Não possuir plano privado de saúde foi associado a menor busca por prevenção. Ser adscrito à ESF não foi associada a busca por ações preventivas. Mesmo com os esforços e com o reconhecimento da importância da prevenção, a maioria dos indivíduos ainda busca os serviços de saúde para tratamento. Palavras-chave Prevenção Primária, Serviços de Saúde, Estratégia de Saúde da Família, Planos de Pré-Pagamento em Saúde, Inquéritos Epidemiológicos 


\section{Introdução}

O conceito de uso dos serviços de saúde está relacionado a um ponto central do funcionamento do mesmo, compreendendo o contato direto consultas diretas com profissionais de saúde ou hospitalização - e indireto - realização de exames complementares preventivos e diagnósticos ${ }^{1}$. Segundo o Modelo Comportamental de Andersen², em sua última versão, o uso dos serviços é dinâmico e influenciado por fatores predisponentes (sociodemográficos, sociais e crenças em saúde); fatores capacitantes (individuais/familiares e a disponibilidade do serviço para uso); necessidades em saúde, sob a ótica do profissional e do próprio usuário; e o sistema de saúde, englobando as políticas de saúde, recursos financeiros e aspectos organizacionais ${ }^{2,3}$.

No Brasil, a comparação de dois inquéritos nacionais, a Pesquisa Nacional por Amostra de Domicílios (PNAD, 2008) e a Pesquisa Nacional de Saúde (PNS, 2013), mostra a manutenção do percentual (14-15\%) de busca pelos serviços de uma forma geral nas duas últimas semanas, mas uma redução da taxa de internação hospitalar nos últimos 12 meses. Além disso, observou-se aumento de $2 \%$ na cobertura por planos privados de saúde, ao passo que a cobertura pela Estratégia de Saúde da Família (ESF) aumentou de $56,1 \%$ para $60,9 \%$ em todo território nacional ${ }^{4}$. Análise de dados da PNAD 2008 observou que indivíduos cadastrados na ESF apresentaram maior probabilidade de terem recebido visita do médico ou dentista nos últimos 12 meses, maior vínculo com os profissionais, maior acesso aos medicamentos necessários e maior satisfação com os serviços de saúde, quando comparados a quem possuía plano privado de saúde e a quem não era cadastrado ${ }^{5}$.

Segundo Starfield ${ }^{6}$, a Atenção Primária à Saúde (APS) deve oferecer à população ações de promoção de saúde, prevenção de agravos, tratamento, diagnóstico e reabilitação, em constante equilíbrio com as necessidades em saúde das populações. Nesse sentido, um dos grandes desafios da ESF, como eixo estruturante da APS, é organizar, dentro de sua agenda, as ações de promoção/prevenção, além das atividades relacionadas ao cuidado clínico, tratamento ou diagnóstico ${ }^{7}$. Portanto, a ESF representa no Brasil o modelo de atenção que busca colocar em prática os princípios discutidos na Conferência de Alma Ata (1978), de valorização da promoção de saúde e prevenção de doenças, gerando um grande desafio para os profissionais e usuários, que devem transferir o foco da cura das doenças para o da prevenção ${ }^{8}$. Os planos privados de saúde também buscam ações preventivas, capazes de reduzir o número de consultas médicas e reduzir os custos com saúde ${ }^{9}$, sendo que o percentual de brasileiros com cobertura dos mesmos aumenta em função da idade, e este grupo tem maiores chances de apresentar comportamento considerados saudáveis capazes de prevenir doenças crônicas ${ }^{8}$.

$\mathrm{Na}$ população brasileira, em pesquisa com dados recordatórios do ano anterior ao momento da coleta, a cada 100 indivíduos, independente da idade e se o serviço utilizado era público ou privado, 71 realizaram consulta médica, 37 realizaram consulta odontológica e 10 foram hospitalizados $^{10}$. Pesquisas realizadas no México, país também em desenvolvimento, como o Brasil, evidenciam que a busca pelos serviços de saúde ainda é predominantemente curativa ${ }^{11}$, apesar de ações de prevenção serem comprovadamente eficazes no combate aos fatores de risco para doenças crônicas ${ }^{12,13}$, reduzindo os custos com internações e consultas especializadas, especialmente quando conduzidas pela ESF${ }^{9}$.

Medina et al. ${ }^{14}$ consideram que ainda são escassos os estudos que tem como desfecho o papel da prevenção e da promoção de saúde dentro do contexto dos serviços de saúde, bem como sobre o papel da APS e da ESF em tais atividades. Diante da importância da participação das atividades de prevenção nos serviços de saúde, especialmente no cenário de predomínio de doenças crônicas, a investigação de fatores associados a essa busca, pode contribuir para o planejamento de ações que visem ampliar o acesso e uso desses serviços. Assim, o objetivo do presente estudo foi analisar a associação entre a busca por serviços preventivos de saúde nas duas últimas semanas e fatores sociodemográficos, posse por plano privado de saúde e cadastro do domicílio na ESF, entre a população adulta participante da PNS (2013). Adicionalmente, avaliou-se se a idade influenciava a possível associação entre o tipo de serviço procurado e a posse de planos de saúde e cadastro na ESF.

\section{Metodologia}

\section{Fonte de dados e amostra}

A Pesquisa Nacional de Saúde (PNS, 2013) é um estudo domiciliar, de abrangência nacional, com objetivo de produzir dados sobre estilo de vida, situação de saúde e atenção à saúde da população brasileira. O plano amostral foi definido 
por conglomerados em três estágios, sendo as unidades primárias de seleção constituídas pelos setores ou conjunto de setores censitários, selecionados por amostragem aleatória simples; os domicílios, também selecionados pelos mesmos critérios utilizados na primeira etapa, constituíram as unidades do segundo estágio; e os moradores adultos, selecionados aleatoriamente com equiprobabilidade entre todos os elegíveis maiores de 18 anos, fizeram parte do terceiro estágio ${ }^{15}$. No presente estudo foram incluídos apenas os moradores adultos (18 anos ou mais) que responderam afirmativamente à pergunta "Nas duas últimas semanas, procurou algum lugar, serviço ou profissional de saúde para atendimento relacionado à própria saúde?”.

\section{Variáveis do estudo}

A variável desfecho do estudo foi definida como o tipo de serviço de saúde procurado ("prevenção" x "tratamento/diagnóstico"), a partir da pergunta "Qual foi o motivo principal pelo qual procurou atendimento relacionado à saúde nas duas últimas semanas?". Foram consideradas “prevenção" ações de "pré-natal”, "puericultura”, "vacinação" e "outro atendimento preventivo". Foram consideradas "tratamento/diagnóstico" as ações "acidente/lesão", "doença”, "problema odontológico", "reabilitação/terapia”, "continuação de tratamento", "parto", "exame complementar diagnóstico", "solicitação de atestado". Aqueles que responderam a opção "outros” foram excluídos da amostra final, pois esta opção não permite definir o uso relatado como preventivo ou curativo/diagnóstico.

As variáveis explicativas incluíram características sociodemográficas, cobertura por plano privado de saúde e ESF. As características sociodemográficas incluíram sexo ("Feminino" ou "Masculino"), idade em anos contínua e dividida por tercis para análise da prevalência, escolaridade ("Até ensino fundamental incompleto"; "Ensino Fundamental Completo até Ensino Médio Incompleto"; "Ensino Médio Completo ou mais"), estado conjugal ("Vive com companheiro" ou "Não vive com companheiro"), cor de pele ("Branca" ou "Não-Branca") e situação censitária do município ("Urbano" ou "Rural"). Foi ainda considerada a posse de plano privado de saúde ("Tem plano de saúde" ou "Não tem plano de saúde") e o cadastro do domicílio na ESF ("não cadastrado"; "cadastrado até 1 ano"; "cadastrado há mais de 1 ano").

\section{Análise estatística}

A descrição das características da amostra foi realizada para todas as variáveis explicativas, considerando a população total e os estratos do desfecho ("prevenção" ou "tratamento/diagnóstico"). A comparação entre esses grupos, sem ajuste, foi realizada pelo teste do Qui-Quadrado, com correção de Rao-Scott, e Teste t para amostras independentes para a variável idade. Adicionalmente, foram calculadas as prevalências e respectivos intervalos de confiança (95\%) para uso de serviços preventivos, segundo as categorias de todas as variáveis explicativas consideradas nesse estudo.

As análises bruta e ajustada da associação entre a busca por serviços preventivos e as variáveis explicativas foram baseadas em Razões de Prevalência (RP), estimadas por meio do modelo de regressão de Poisson com variância robusta. Foram testadas interação entre a variável idade e as variáveis posse de planos de saúde e cadastro na ESF. Foi ainda feita análise de sensibilidade com a exclusão dos indivíduos que relataram a opção de uso de serviço "Exames Complementares Diagnósticos" da amostra, considerando que esse grupo poderia ser alocado nas categorias de prevenção ou tratamento, visando avaliar o impacto dessa categoria nos principais resultados descritos no presente estudo.

Os gráficos das probabilidades preditas de busca por serviço preventivos em função da idade foram construídos a partir de um modelo de Regressão Logística, considerando os ajustes pelas mesmas variáveis incluídas no modelo de Poisson.

As análises foram realizadas através do pacote estatístico Stata versão 14.0 (Stata Corp LLP, College Station, TX), considerando a complexidade da amostra.

\section{Considerações éticas}

A PNS foi aprovada pelo Comitê Nacional de Ética em Pesquisa com Seres Humanos (Conep).

\section{Resultados}

A amostra total da PNS é composta por 205.529 indivíduos em todo território nacional. Destes, $36.036(17,54 \%)$ relataram ter procurado o serviço de saúde nas duas últimas semanas. Foram excluídos 3.659 (10,15\%) que responderam "outros" em relação ao motivo principal para a bus- 
ca do serviço, compondo então a amostra deste estudo um total de 32.376 sujeitos. Dentre estes, $28.187(87,06 \%$ - IC95\% 85,78-88,20) relataram motivo da busca pelo serviço classificado como "tratamento/diagnóstico" e 4.189 (12,94\% IC95\% 11,7-14,2) como "prevenção".

A Tabela 1 apresenta a descrição da amostra juntamente com a análise univariada para comparação entre a distribuição das variáveis explicativas e o tipo de serviço de saúde procurado pelos indivíduos (preventivo ou tratamento/diagnóstico). A amostra é composta em sua maioria por mulheres $(64,72 \%)$ com idade média de 47,99 $( \pm 0,35)$. Somente em relação a cor da pele, situação censitária do domićlio e cadastro na ESF não houve diferença entre quem procurou serviços preventivos ou tratamento. Para as demais variáveis (sexo, idade, estado civil, escolaridade, posse de plano privado de saúde) houve associação com o serviço procurado $(\mathrm{p}<0,05)$.
A Tabela 2 mostra a prevalência de uso de serviços preventivos, com seus respectivos Intervalos de Confiança (IC95\%) para cada categoria das variáveis explicativas incluídas no estudo. Para definição destas categorias, a idade foi categorizada em 3 faixas etárias, por tercis.

A Tabela 3 apresenta o resultado da análise bruta e ajustada para associação entre o tipo de serviço procurado e as variáveis explicativas incluídas na análise. Após ajustamento por todas as variáveis, as mulheres tiveram maior probabilidade de procurar ações preventivas, ao passo que indivíduos mais velhos, que não viviam com companheiro e que não tinham plano de saúde apresentaram menor probabilidade de buscar as mesmas ações. $\mathrm{O}$ cadastro na ESF não foi associado a maior ou menor probabilidade de buscar medidas de prevenção em saúde. As interações testadas entre idade e as variáveis posse de plano privado de saúde e cadastro na ESF não foram

Tabela 1. Características da amostra total estudada e segundo tipo de serviço usado. Pesquisa Nacional de Saúde (2013).

\begin{tabular}{|c|c|c|c|c|}
\hline \multirow{2}{*}{ Variáveis } & \multirow{2}{*}{ Amostra Total } & \multicolumn{2}{|c|}{ Tipo de serviço } & \multirow{2}{*}{$\begin{array}{c}\text { Valor } \\
\mathbf{p}^{*}\end{array}$} \\
\hline & & Tratamento & Prevenção & \\
\hline Sexo $(\%)$ & & & & $<0,001$ \\
\hline Feminino & 64,72 & 54,70 & 10,02 & \\
\hline Masculino & 35,28 & 32,36 & 2,92 & \\
\hline Idade em anos, média (desvio padrão) & $47,99(0,35)$ & $39,24(0,15)$ & $32,65(0,35)$ & $<0,001$ \\
\hline Cor da pele $(\%)$ & & & & 0,120 \\
\hline Branca & 51,24 & 44,14 & 7,10 & \\
\hline Não branca & 48,73 & 42,92 & 5,83 & \\
\hline Estado civil (\%) & & & & 0,006 \\
\hline Vive com companheiro & 63,33 & 54,35 & 8,98 & \\
\hline Não vive com companheiro & 36,67 & 32,71 & 3,96 & \\
\hline Escolaridade (\%) & & & & $<0,001$ \\
\hline Até fundamental completo & 49,05 & 39,18 & 4,37 & \\
\hline Fundamental completo até médio incompleto & 15,65 & 11,68 & 1,47 & \\
\hline Médio completo ou mais & 35,30 & 3,62 & 7,09 & \\
\hline \multicolumn{5}{|l|}{ Localização do domicílio (\%) } \\
\hline Urbana & 81,04 & 77,64 & 11,58 & 0,835 \\
\hline Rural & 18,96 & 9,42 & 1,36 & \\
\hline Possui plano privado de saúde (\%) & & & & $<0,001$ \\
\hline Sim & 36,71 & 30,82 & 5,89 & \\
\hline Não & 63,29 & 56,25 & 7,05 & \\
\hline Cadastro do domicílio na ESF (\%) & & & & 0,502 \\
\hline Não & 43,81 & 37,89 & 5,92 & \\
\hline Sim, até 1 ano & 7,56 & 6,49 & 1,07 & \\
\hline Sim, acima de 1 ano & 48,63 & 42,68 & 5,95 & \\
\hline
\end{tabular}

Tratamento/diagnóstico: acidente/lesão, doença, problema odontológico, reabilitação/terapia, continuação de tratamento, parto, exame complementar diagnóstico e solicitação de atestado. Prevenção: pré-natal, puericultura, vacinação e outro atendimento preventivo. ESF: Estratégia de Saúde da Família. ${ }^{\star}$ Teste do Qui-Quadrado com correção de Rao-Scott ou Teste t de Student. 
Tabela 2. Prevalência da busca por serviços preventivos, segundo variáveis exploratórias. Pesquisa Nacional de Saúde (2013).

\begin{tabular}{|c|c|c|}
\hline Variáveis & $\begin{array}{l}\text { Prevalência de busca por } \\
\text { serviços de prevenção }\end{array}$ & Intervalo de confiança (95\%) \\
\hline \multicolumn{3}{|l|}{ Sexo } \\
\hline Feminino & 15,47 & $13,86-17,24$ \\
\hline Masculino & 8,28 & $6,77-10,08$ \\
\hline \multicolumn{3}{|l|}{ Faixa etária em anos ${ }^{\star \star}$} \\
\hline $18-38$ & 16,81 & $12,03-22,97$ \\
\hline $39-56$ & 17,27 & $15,01-19,79$ \\
\hline 57 ou mais & 10,26 & $8,94-11,74$ \\
\hline \multicolumn{3}{|l|}{ Cor da Pele } \\
\hline Branca & 13,86 & $12,04-15,90$ \\
\hline Não branca & 11,96 & $10,55-13,54$ \\
\hline \multicolumn{3}{|l|}{ Estado civil } \\
\hline Vive com companheiro & 14,18 & $12,63-15,89$ \\
\hline Não vive com companheiro & 10,78 & $9,14-12,68$ \\
\hline \multicolumn{3}{|l|}{ Escolaridade } \\
\hline Até fundamental completo & 10,04 & $8,42-11,92$ \\
\hline Fundamental completo/Médio Incompleto & 11,20 & $8,47-14,66$ \\
\hline Médio completo ou mais & 1,63 & $1,44-1,85$ \\
\hline \multicolumn{3}{|l|}{ Localização do domicílio } \\
\hline Urbano & 12,97 & $11,69-14,37$ \\
\hline Rural & 12,62 & $9,87-15,99$ \\
\hline \multicolumn{3}{|l|}{ Possui plano privado de saúde } \\
\hline Sim & 16,05 & $13,92-18,43$ \\
\hline Não & 11,13 & $9,73-12,69$ \\
\hline \multicolumn{3}{|l|}{ Cadastro do domicílio na ESF } \\
\hline Não & 13,51 & $11,79-15,43$ \\
\hline Sim, até 1 ano & 14,09 & $10,4-18,72$ \\
\hline Sim, acima de 1 ano & 12,23 & $10,55-14,15$ \\
\hline
\end{tabular}

significativas dentro do modelo de Regressão de Poisson ( $\mathrm{p}>0,05)$.

A análise de sensibilidade, conduzida para avaliar o impacto da exclusão da categoria "Exames Complementares Diagnósticos” nos resultados apresentados na Tabela 3, mostrou que as associações se mantiveram na mesma direção, significância e com magnitudes semelhantes, sendo essa categoria, portanto, mantida no grupo denominado "Tratamento/diagnóstico".

A Figura 1 apresenta a probabilidade predita do indivíduo buscar o serviço de saúde para prevenção, segundo a posse de plano de saúde e cadastro na ESF. A probabilidade predita de busca por serviços preventivos é maior entre os mais jovens, independente da posse ou não de plano privado de saúde e essa busca também é maior em quem tem plano, o que se mantém em todas as idades. Para o cadastro na ESF, a probabilidade predita de busca por serviços preventivos é a mesma para todas as categorias dessa variável, mantendo a diferença em relação à idade.

\section{Discussão}

O presente estudo mostrou que quase um quinto da população adulta brasileira procurou algum serviço de saúde nas duas semanas anteriores à entrevista, com 12,94\% desses buscando por serviços classificados como preventivos. Além disso, os dados ressaltaram que a procura por serviços preventivos foi mais frequente entre as mulheres e menos frequente entre os mais velhos, sem companheiro e sem plano privado de saúde. Adicionalmente, o cadastro do domicílio na ESF 
Tabela 3. Associação entre a busca por serviços preventivos de saúde e as variáveis explicativas. Pesquisa Nacional de Saúde (2013).

\begin{tabular}{|c|c|c|}
\hline Variáveis & $\begin{array}{c}\text { Razão de Prevalência bruta } \\
\text { (Intervalo de } \\
\text { confiança-95\%) }\end{array}$ & $\begin{array}{c}\text { Razão de Prevalência } \\
\text { ajustada }^{\star} \text { (intervalo de } \\
\text { confiança-95\%) }^{\text {con }}\end{array}$ \\
\hline \multicolumn{3}{|l|}{ Sexo } \\
\hline Masculino & 1,00 & 1,00 \\
\hline Feminino & $1,86(1,49-2,34)$ & $1,87(1,49-2,34)$ \\
\hline Idade em anos & $0,98(0,97-0,99)$ & $0,98(0,97-0,99)$ \\
\hline \multicolumn{3}{|l|}{ Cor da pele } \\
\hline Branca & 1,00 & 1,00 \\
\hline Não branca & $0,86(0,71-1,03)$ & $0,87(0,72-1,06)$ \\
\hline \multicolumn{3}{|l|}{ Estado civil } \\
\hline Vive com companheiro & 1,00 & 1,00 \\
\hline Não vive com companheiro & $0,76(0,62-0,92)$ & $0,72(0,59-0,88)$ \\
\hline \multicolumn{3}{|l|}{ Escolaridade } \\
\hline Até fundamental incompleto & 1,00 & 1,00 \\
\hline Fundamental Completo a médio incompleto & $1,11(0,80-1,55)$ & $0,95(0,66-1,36)$ \\
\hline Médio completo ou mais & $1,63(1,31-2,02)$ & $1,20(0,92-1,57)$ \\
\hline \multicolumn{3}{|l|}{ Localização do município } \\
\hline Urbano & 1,00 & 1,00 \\
\hline Rural & $0,97(0,74-1,26)$ & $1,13(0,86-1,50)$ \\
\hline \multicolumn{3}{|l|}{ Posse de plano privado de saúde } \\
\hline Sim & 1,00 & 1,00 \\
\hline Não & $0,69(0,56-0,84)$ & $0,76(0,62-0,94)$ \\
\hline \multicolumn{3}{|l|}{ Cadastro na ESF } \\
\hline Não & 1,00 & 1,00 \\
\hline Sim, até de 1 ano & $1,04(0,75-1,43)$ & $1,07(0,77-1,51)$ \\
\hline Sim, acima de 1 ano & $0,90(0,74-1,10)$ & $0,96(0,79-1,17)$ \\
\hline
\end{tabular}

ESF: Estratégia de Saúde da Família. ${ }^{*}$ Ajustadas pelas variáveis listadas na tabela, estimadas pela Regressão de Poisson com variância robusta.

não apresentou associação com o tipo de serviço demandado pela população.

A busca por serviços de saúde nas últimas duas semanas foi relatada por $17,54 \%$ dos indivíduos, sendo superior às registradas nas Pesquisas Nacionais por Amostra de Domicílios (PNAD) de 2003 (14,6\%) e $2008(14,6 \%)^{4}$. Além disso, apenas uma pequena parcela da população $(12,94 \%)$ que buscou os serviços de saúde nas duas últimas semanas o fez para ações de prevenção. Em 2003, a procura por esse mesmo motivo foi registrada para $31,3 \%$ e em 2008 por $24,7 \%$ da população adulta brasileira ${ }^{16}$, indicando possível redução dessa proporção com o passar dos anos. Esta redução está em desacordo com o que é preconizado no Plano de Ação para Prevenção e Controle de Doenças não Transmissíveis (DCNT, 2011-2022) que, entre suas ações e metas, visa ao controle dos fatores de risco e prevenção das DCNT, ressaltando a importância dos cuidados preventivos ${ }^{17}$. Um estudo realizado com a população mexicana demonstrou que indivíduos submetidos a um programa de prevenção de doenças crônicas alcançou redução de alguns fatores de risco como Índice de Massa Corporal (IMC), circunferência de cintura e quadril, peso e taxas de colesterol e triglicerídeos ${ }^{12}$, ressaltando a importância desse contato com os serviços e o possível impacto negativo da baixa prevalência de indivíduos que busca os serviços preventivos, como demonstrado na presente análise. Estudo também realizado com dados da PNS 2013 demonstrou que indivíduos que possuíam doenças crônicas realizaram 1,26 mais consultas médicas quando comparados aqueles que não apresentavam essas doenças ${ }^{18}$. A categoria "consulta médica" foi considerada como "tratamento" neste estudo, o que mostra que mesmo com a tentativa de incentivo à prevenção, a maioria dos indivíduos ainda buscam o serviço para tratamento, considerando 

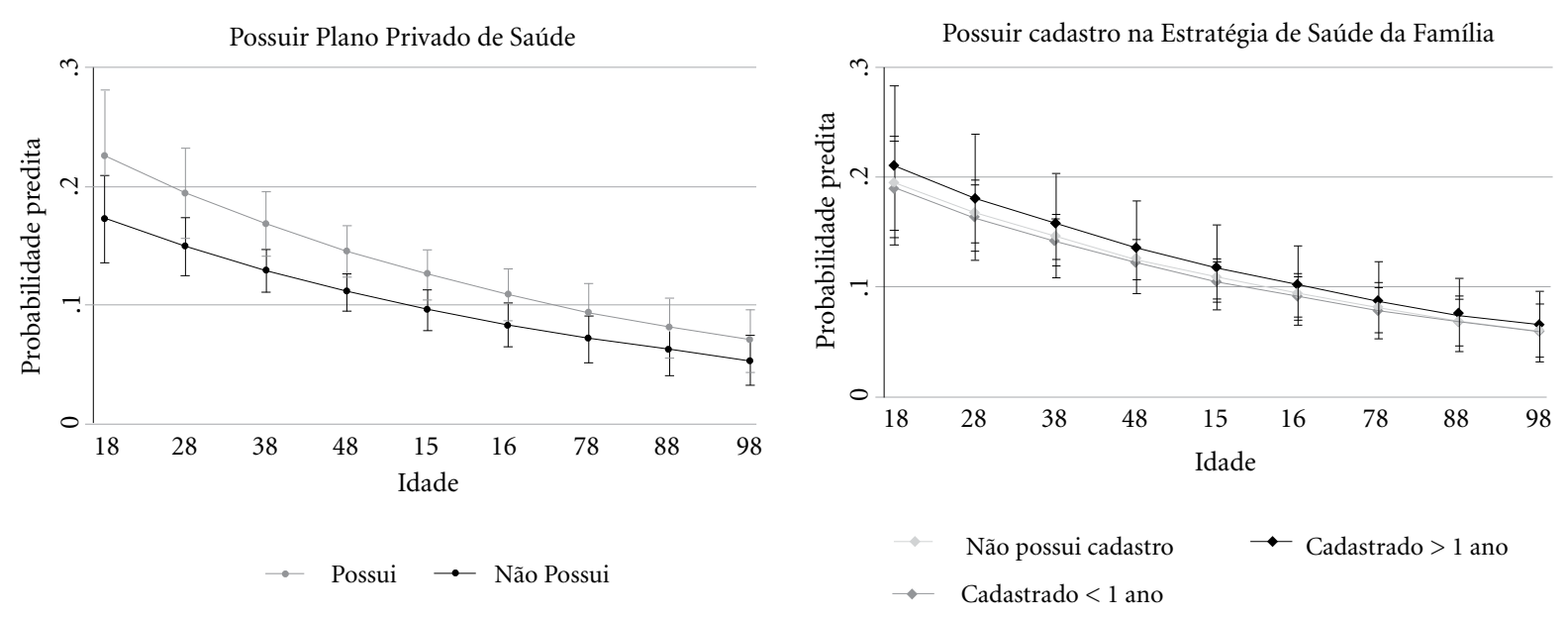

Figura 1. Probabilidade Predita de busca por serviços preventivos em função da idade, em relação a possuir planos de saúde e cadastro na Estratégia de Saúde da Família.

que na PNS 2013, 45,1\% dos indivíduos possuem diagnóstico de pelo menos uma DCNT ${ }^{19}$.

As mulheres apresentaram maior probabilidade de buscarem serviços ligados à prevenção, em comparação à população masculina. Este resultado já foi observado na PNAD de 1998, quando um percentual maior de mulheres procurou serviços de saúde classificados como preventi$\operatorname{vos}^{20}$, o que está de acordo com o fato das mulheres terem a tendência a se preocupar mais com a saúde que os homens. Como o objetivo central do presente estudo não era a verificação da diferença dos gêneros em relação a busca por prevenção ou tratamento, o "pré-natal" foi inserido como ação preventiva, o que também contribuiu para maior probabilidade da busca por serviços preventivos entre o sexo feminino. Ainda assim, esses resultados reforçam a necessidade de estratégias que visem aumentar o acesso aos serviços de saúde para a população masculina, sobretudo os preventivos, como já estabelecido nas políticas nacionais ${ }^{21}$.

O percentual de indivíduos que buscaram serviços preventivos foi menor entre os mais velhos. Este resultado pode ser considerado negativo em relação a diversos aspectos da saúde do idoso, uma vez que idosos que apresentam perfis de uso de serviços de saúde predominantemente preventivos apresentam melhor qualidade de vida, especialmente entre aqueles com condições crônicas ${ }^{11}$. De forma contrária, idosos com limitação em atividades de vida diária utilizam o serviço de saúde de forma predominantemente curativa, mesmo em Unidades Básicas de Saúde ${ }^{22}$. Este estudo não analisou questões funcionais referentes aos idosos da amostra, mas a redução da busca por ações preventivas em faixas etárias mais elevadas pode ser devido a maior limitação funcional quando comparado a indivíduos mais jovens, e então maior procura por consultas médicas, continuidade de tratamentos longos e reabilitação.

No presente estudo, indivíduos que não viviam com o companheiro apresentaram menor proporção de busca por serviços preventivos na última semana. Viver com o companheiro é um item relacionado ao capital social do indivíduo, o que pode falar a favor de maior suporte para busca por serviços preventivos. No estudo de Fang et al. ${ }^{23}$, apresentar capital social positivo foi associado a melhor adesão à atividade física e alimentação saudável, consideradas ações preventivas em saúde, o que reforça as evidências relatadas no presente estudo.

Em relação a planos privados de saúde, na amostra do presente estudo, $36,71 \%$ dos indivíduos possuíam essa cobertura, evidenciando um aumento desse percentual quando comparado aos dados das PNADs de $2003(24,6 \%)$ e 2008 $(25,9 \%)^{4}$. A posse por esses planos aumentou a probabilidade de procura por serviços preventivos e essa diferença se manteve ao longo de todas as idades. Sabe-se que uma maior proporção de indivíduos que possuem planos de saúde tende a apresentar alimentação mais saudável, maior proporção de prática de exercício físico durante o tempo livre e realização de mamografia e Papanicolau, demonstrando que essa população apre- 
senta um perfil de maior cuidado com a saúde ${ }^{8}$, estando coerente com os resultados descritos na presente análise.

A adesão à programas de prevenção de agravos e promoção de saúde levou à redução do número de consultas médicas e internação em 4 anos, gerando menores custos para o prestador de serviços ${ }^{9}$. O presente estudo não avaliou os custos em saúde, mas seus resultados são semelhantes em relação a maior adesão de quem tem plano de saúde às ações preventivas, falando a favor do possível impacto positivo nos custos da atenção à saúde com o aumento da busca por serviços de prevenção e promoção da saúde.

O estudo de Macinko e Lima-Costa ${ }^{5}$ discorre sobre um perfil de uso dos serviços por indivíduos com baixa escolaridade e que não possuem planos de saúde de menor busca por serviços preventivos, uma vez que os com menor escolaridade tem menor renda e consequentemente, menor chance de possuir plano privado de saúde ${ }^{5}$. Entre a escolaridade e a posse de planos de saúde, no presente estudo, provavelmente os planos de saúde tem maior influência no tipo de serviço buscado, pois sua associação com o desfecho é mantida na análise ajustada, o que não acontece com a escolaridade. Ao mesmo tempo, este mesmo grupo é aquele que mais usa os serviços da ESF, e deveria ter mais acesso a ações de prevenção e promoção de saúde, o que não é corroborado pelos resultados do presente estudo.

O cadastro na Estratégia de Saúde da Família (ESF) há mais de um ano foi observado em $48,63 \%$ dos domicílios da amostra, além de ter sido observado que 7,56\% estavam cadastrados há menos de um ano. Quando comparado a PNAD de 2008, onde foi registrado 50,9\% de cobertura, independente do tempo, observa-se aumento em todo território nacional, especialmente entre a população com menor nível de escolaridade $^{18}$. Considerando ser uma estratégia fundamental como garantia de saúde integral, baseada na equidade, coordenação do cuidado e longitudinalidade ${ }^{6}$, esta ampliação da cobertura é relevante, embora ainda esteja inferior a de outros países em desenvolvimento. A Costa Rica, por exemplo, apresenta $88 \%$ de cobertura do programa EBAIS (Equipos Básicos de Atención Integral en Salud), semelhante à ESF brasileira ${ }^{24}$, e Cuba, cujo sistema de saúde é inteiramente coberto por recursos públicos, consegue cobrir $100 \%$ da população ${ }^{25}$. O "National Health Service” (NHS) da Inglaterra, maior sistema em redes inteiramente financiado pelo governo federal, apresenta cobertura de $100 \%$ da população, sen- do mais utilizada nas zonas menos urbanizadas, com menor nível de desenvolvimento e entre a população acima de $60 \operatorname{anos}^{26}$.

No presente estudo, ter o domicílio cadastrado na ESF não foi associado ao tipo de serviço de saúde procurado, independente das demais variáveis consideradas. Um grande desafio para as ESF é a conciliação dos serviços de prevenção e promoção de saúde, preconizados por portarias e projetos das Secretarias Municipais e Estaduais de Saúde, uma vez que a maioria das equipes ainda atua com características essencialmente assistenciais ${ }^{27}$. Uma pesquisa com dados do Programa Nacional de Melhoria do Acesso e da Qualidade da Atenção Básica (PMAQ-AB) evidencia que, em todo o Brasil, as ações de prevenção realizadas na APS são incipientes e inferiores ao esperado das equipes, especialmente aquelas direcionadas aos idosos ${ }^{14}$. Essas informações corroboram os achados do presente estudo, quando a busca por ações preventivas não foi associada ao cadastramento nas ESF. No Brasil, ainda falta à APS incorporar práticas preventivas na rotina dos serviços, como em outros países que contam com reformas ligadas ao fortalecimento deste nível de atenção, como observado na Espanha ${ }^{28}$.

Cabe ressaltar que atividades como "vacinação" e "pré-natal", classificadas como preventivas no presente estudo, estão presentes em todas as áreas cobertas pela ESF, independente do tempo de cobertura. No entanto, ainda assim, a inclusão dessa informação não foi suficiente para aumentar a proporção de busca por serviços preventivos nas áreas cobertas. O Programa Nacional de Imunização coloca a vacinação como ação prioritária para a APS e a maioria das Unidades Básicas de Saúde tem salas de vacina funcionando e consideradas boas ou ótimas, embora existam barreiras relacionadas ao horário de funcionamento e à divulgação da importância da vacinação, em todas as faixas etárias ${ }^{29}$. Em relação ao pré-natal, as consultas fazem parte da agenda, especialmente da enfermagem, e é vista pelas gestantes de forma positiva, como momento de acolhimento, retirada de dúvidas, espaço educativo e importante para o estabelecimento de vínculos ${ }^{30}$. A "puericultura”, atividade também considerada "prevenção" no presente estudo, ao contrário, já não é fortemente consolidada entre as ações da agenda da ESF, sendo os cuidados com o recémnascido pré-termo não considerado como ação da APS e focado no modelo biomédico, com importantes deficiências no processo ${ }^{31,32}$. Portanto, estudos que busquem entender os motivos da não associação entre a existência de cobertura 
pela ESF e a busca por serviços preventivos podem auxiliar no direcionamento de políticas que visem incorporar, efetivamente, essas ações na rotina das atividades desenvolvidas pelas equipes.

A PNS 2013 constitui uma amostra de base populacional, com representatividade nacional, o que confere robustez aos resultados apresentados sobre a relação entre a procura por serviços de saúde de forma preventiva e os fatores associados. São limitações do estudo aquelas inerentes aos inquéritos transversais e também a falta de informações mais detalhadas em relação a opções de busca por serviços de saúde para ações de prevenção, especialmente as específicas para DCNT e estímulo à prática de atividade física. É possível que algumas ações ligadas à prevenção e promoção de saúde sejam realizadas, especial- mente pelas equipes da ESF e não tenham sido registradas devido à forma das perguntas do inquérito, o que pode ter diluído as forças das associações descritas nesse estudo.

Entretanto, nossos resultados permitiram retratar os fatores associados à busca por ações preventivas, permitindo uma caracterização da população com maior probabilidade de procura por esse tipo de serviço. Além dos fatores sociodemográficos, chama atenção que ter planos de saúde aumentou as possibilidades de procura por esse tipo de serviço, enquanto o cadastro do domicílio na ESF não se associou a essa procura. Esses resultados podem sugerir a necessidade de maior capacitação e estímulo das equipes da ESF para incorporação de ações de prevenção em suas agendas, incentivando sua prática na população.

\section{Colaboradores}

SLA Silva foi responsável pelo delineamento da análise, seleção das variáveis e definição dos desfechos, construção da variável de desfecho e das co-variáveis, realização das análises no programa estatístico STATA, interpretação dos resultados, revisão da literatura e escrita do documento. JL Torres foi responsável pela construção das variáveis desfecho e das co-variáveis, realização das análises no programa estatístico STATA, interpretação dos resultados, revisão do texto final. SV Peixoto foi responsável pelo delineamento da análise, seleção das variáveis e definição dos desfechos, fornecimento dos dados da PNS para análise, revisão do texto final.

\section{Referências}

1. Travassos C, Martins M. Uma revisão sobre os conceitos de acesso e utilização de serviços de saúde. Cad Saude Publica 2004; 20(2):190-198.

2. Andersen RM. Revisiting the behavioral model and access to medical care: does it matter? JHSB 1995; 36(1):1-10.

3. Pavão ALB, Coeli CM. Modelos teóricos do uso de serviços de saúde: conceitos e revisão. Cad Saude Colet 2008; 16(3):471-482.

4. Viacava F, Bellido JG. Condições de saúde, acesso a serviços e fontes de pagamento, segundo inquéritos domiciliares. Cien Saude Colet 2016; 21(2):351-370.

5. Macinko J, Lima-Costa MF. Access to, use of and satisfaction with health services among adults enrolled in Brazil's Family Health Strategy: evidence from the 2008 National Household Survey. TMIJ 2012; (1):3642 .

6. Starfield B. Atenção Primária: equilíbrio entre necessidades de saúde, serviços e tecnologia. Brasília: UNESCO, MS; 2002.

7. Norman AH, Tesser CD. Acesso ao cuidado na Estratégia Saúde da Família: equilíbrio entre demanda espontânea e prevenção/promoção da saúde. Saude Soc $2015 ; 24(1): 165-179$.

8. Malta DC, Bernal RTI. Comparação dos fatores de risco e proteção de doenças crônicas na população com e sem planos de saúde nas capitais brasileiras, 2011. Rev Bras Epidemiol 2014; 17(1):241-225. 
9. Alves DC, Ugá MAD, Portela MC. Promoção da saúde, prevenção de doenças e utilização de serviços: avaliação das ações de uma operadora de plano de saúde brasileira. Cad Saude Colet 2016; 24(2):153-161.

10. Araujo MEA, Silva MT, Andrade KRC, Galvão TF, Pereira MG. Prevalência de Utilização de Serviços de Saúde no Brasil: revisão sistemática e metanálise. Epidemiol Serv Saude 2017; 26(3):589-604.

11. Carrillo KG, García-Peña C, Duran-Muñoz C, Mudgal J, Duran-Arenas L, Salmerón-Castro J. Health care utilization and health-related quality of life perception in older adults: a study of the Mexican Social Security Institute. Salud Publica Mex 2008; 50(3):207217.

12. Denman CA, Rosales C, Cornejo E, Bell ML, Munguía D, Zepeda T, Carvajal S, Zapien JG. Evaluation of the Community-Based Chronic Disease Prevention Program Meta Salud in Northern Mexico, 2011-2012. Prev Chronic Dis 2014; 11:E154.

13. Hurtado M, Spinner JR, Yang M, Evensen C, Windham A, Ortiz G, Tracy R, Ivy ED. Knowledge and Behavioral Effects in Cardiovascular Health: Community Health Worker Health Disparities Initiative, 2007-2010. Prev Chronic Dis 2014; 11:E22.

14. Medina MG, Aquino R, Vilasbôas ALQ, Mota E, Júnior EPP, Luz LA, Anjos DSO, Pinto ICM. Promoção da saúde e prevenção de doenças crônicas: o que fazem as equipes de Saúde da Família? Saúde Debate 2014; 38(n. esp.):69-82.

15. Souza-Junior PRB, Freitas MPS, Antonassi GA, Szwarcwald CL. Desenho da amostra da Pesquisa Nacional de Saúde 2013. Epidemiol Serv Saude 2015; 24(2):207-216.

16. Silva ZP, Ribeiro MCSA, Barata RB, Almeida MF Perfil sociodemográfico e padrão de utilização dos serviços de saúde do Sistema Único de Saúde (SUS), 20032008. Cien Saude Colet 2011; 16(9):3807-3816.

17. Ministério da Saúde (MS). Plano de Ações Estratégicas para o Enfrentamento de Doenças Crônicas Não Transmissiveis (DCNT) no Brasil (2011-2020) [documento na Internet]. Brasília: MS; 2001. [acessado 2017 Maio 21]. Disponível em: http://bvsms.saude.gov.br/bvs/ publicacoes/plano_acoes_enfrent_dcnt_2011.pdf

18. Malta DC, Santos MAS, Stoppa SR, Vieira JEB, Melo EA, Reis AAC. A Cobertura da Estratégia de Saúde da Família (ESF) no Brasil, segundo a Pesquisa Nacional de Saúde, 2013. Cien Saude Colet 2016; 21(2):327-338.

19. Malta DC, Stopa SR, Szwarcwald CL, Gomes NL, Silva Júnior JB, Reis AAC. A vigilância e o monitoramento das principais doenças crônicas não transmissíveis no Brasil - Pesquisa Nacional de Saúde, 2013. Epidemiol Serv Saude 2015; 18(Supl. 2):3-16.

20. Pinheiro RS, Viacava F, Travassos C, Brito AS. Gênero, morbidade, acesso e utilização de serviços de saúde no Brasil. Cien Saude Colet 2002; 7(4):687-707.

21. Ministério da Saúde (MS). Política Nacional de Atenção Integral à Saúde do Homem 2008 [documento na Internet]. Brasília: MS; 2008. [acessado 2017 Maio 21]. Disponível em: http://bvsms.saude.gov.br/ bvs/publicacoes/politica_nacional_atencao_saude_ homem
22. Silva AMM, Mambrini JVM, Peixoto SV, Malta DC, Lima-Costa MF. Uso de serviços de saúde por idosos brasileiros com e sem limitação funcional. Rev Saude Publica 2017; 51(Supl. 1):5s.

23. Fang J, Wang J, Li J, Li H, Shao C. The correlates of social capital and adherence to healthy lifestyle in patients with coronary heart disease. Patient Prefer Adherence 2017; 11:1701-1707.

24. Soors W, Paepe P, Unger J. Management commitments and primary care: another lesson from costa rica for the world? Int J Health Serv 2014; 44(2):337-353.

25. Domínguez-Alonso E, Zacca E. Sistema de salud de Cuba. Salud Publica de Méx 2011; 53(2):168-176.

26. Burch P, Doran T, Kontopantelis E. Regional variation and predictors of over-registration in English primary care in 2014: a spatial analysis. J Epidemiol Community Health 2018; 72(6):532-538.

27. Beato MSF, Stralen CJ, Passos ICF. A discursive analysis of health promotion meanings incorporated into the Family Health Strategy. Interface (Botucatu) 2011; 15(37):529-537.

28. March S, Martín MJ, Gomis IM, Azagra CBB, Soto LE, Ramos M. ¿Qué hacemos en el barrio? Descripción de las actividades comunitarias de promoción de la salud en atención primaria: Proyecto frAC. Gac Sanit 2014; 28(4):267-273.

29. Siqueira LG, Martins AMEBL, Versiani CMC, Almeida LAV, Oliveira CS, Nascimento JE, Alecrim BPA, Bezerra RC. Avaliação da organização e funcionamento das salas de vacina na Atenção Primária à Saúde em Montes Claros, Minas Gerais, 2015. Epidemiol Serv Saude 2017; 26(3):557-568.

30. Landerdahl MC, Ressel LB, Martins FB, Bheregaray F, Gonçalves CMO. A percepção de mulheres sobre atenção pré-natal em uma Unidade Básica de Saúde. Esc Anna Nery 2007; 11(1):105-111.

31. Aires LCP, Santos EKA, Costa R, Borck M, Custódio ZAO. Seguimento do bebê na atenção básica: interface com a terceira etapa do método canguru. Rev Gaúcha de Enferm 2015; 36(n. esp.):224-232.

32. Pedraza DF, Santos IS. Avaliação da vigilância do crescimento nas consultas de puericultura na Estratégia Saúde da Família em dois municípios do estado da Paraíba, Brasil. Epidemiol Serv Saude 2017; 26(4):847855.

Artigo apresentado em 14/03/2018

Aprovado em 19/06/2018

Versão final apresentada em 21/06/2018 